\title{
Reciprocal Frame Structures Made Easy
}

Peng Song* Chi-Wing Fu* Prashant Goswami Jianmin Zheng Nanyang Technological University, Singapore
Niloy J. Mitra

University College London
Daniel Cohen-Or

Tel Aviv University
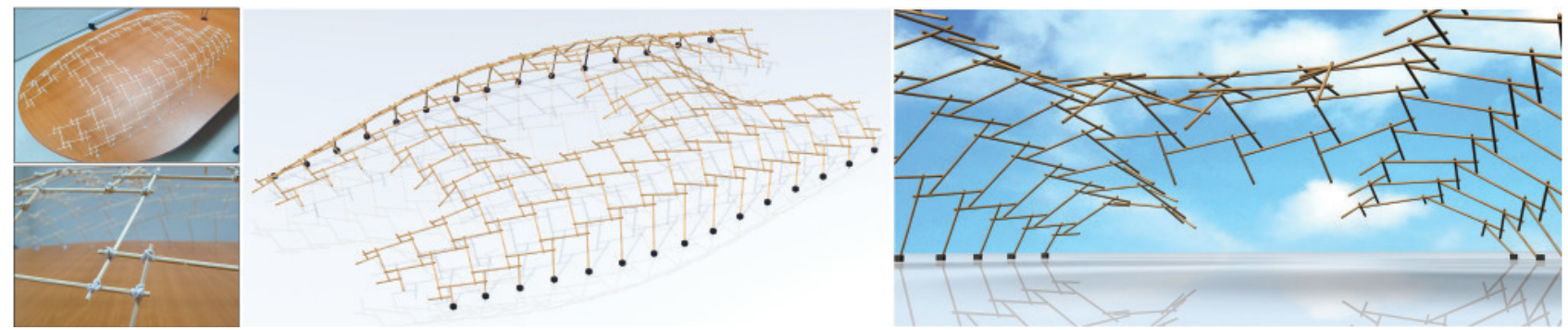

Figure 1: A large reciprocal frame $(R F)$ structure designed by our method; there are no central supports as rods rest on and are supported by adjacent rods reciprocally. When constructed, rods are usually tied or nailed, as shown on the left, in a physical model that we built.

\section{Abstract}

A reciprocal frame $(\mathrm{RF})$ is a self-supported three-dimensional structure made up of three or more sloping rods, which form a closed circuit, namely an RF-unit. Large RF-structures built as complex grillages of one or a few similar RF-units have an intrinsic beauty derived from their inherent self-similar and highly symmetric patterns. Designing RF-structures that span over large domains is an intricate and complex task. In this paper, we present an interactive computational tool for designing RF-structures over a 3D guiding surface, focusing on the aesthetic aspect of the design.

There are three key contributions in this work. First, we draw an analogy between RF-structures and plane tiling with regular polygons, and develop a computational scheme to generate coherent RF-tessellations from simple grammar rules. Second, we employ a conformal mapping to lift the 2D tessellation over a 3D guiding surface, allowing a real-time preview and efficient exploration of wide ranges of RF design parameters. Third, we devise an optimization method to guarantee the collinearity of contact joints along each rod, while preserving the geometric properties of the RF-structure. Our tool not only supports the design of wide variety of RF pattern classes and their variations, but also allows preview and refinement through interactive controls.

CR Categories: I.3.5 [Computational Geometry and Object Modeling]: Curve, surface, solid, and object representations;

Keywords: Reciprocal frame, computational design

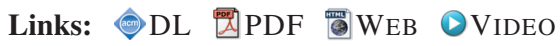

* joint first authors

\section{Introduction}

The reciprocal frame (RF) is a three-dimensional assembly structure made up of three or more sloping rods in a closed circuit, namely an $R F$-unit (see Figure 2 (left)). The inner end of each rod rests on and is supported by its adjacent rod. Signified by the word "reciprocal," which expresses mutual action or relationship, such closed circuit is obtained as the last rod is placed over the first one in a mutually-supporting manner. At the outer end, the rods are given an external support by a wall, ring beams, or columns.

The fundamental concept of reciprocal frames [Chilton 1995; Larsen 2008], has been known for many centuries. One classical example is the various architectural designs by Leonardo Da Vinci during the Renaissance. However, it was only until the very recent decades that this topic caught the attention of architects and structural engineers, because of the emerging applications of computational optimization and CAD tools to enhance, enrich, and scale up the construction [Pugnale et al. 2011].

The reciprocal frames are fascinating. Starting with only very simple material in the form of rods, one can build a complex grillage structure made of one or a few similar RF-units (see Figure 1), by iteratively putting RF-units around one another [Bertin 2001]. No central supports are required in the resulting RF-structures, and one can also disassemble and re-assemble these structures, facilitating their transportation from place to place. This makes RF a highly cost-effective deployable system, particularly suitable for rapid constructions of temporary structures [Larsen 2008].

Apart from the technical aspects, the reciprocal frames also have their intrinsic beauty. Similar to bird nests in the nature, which are built from discrete simple elements, the reciprocal frames share a common characteristic of being a modular structure composed with simple rods. These rods nicely form self-similar and highly symmetric patterns, capable of creating a vast architectural space as a narrative and aesthetic expression of the building.
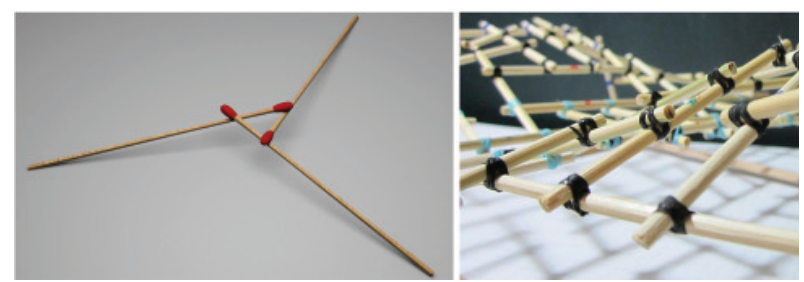

Figure 2: Left: A three rods reciprocal frame, as an RF-unit. Right: a large RF-structure made up of tied rods. 


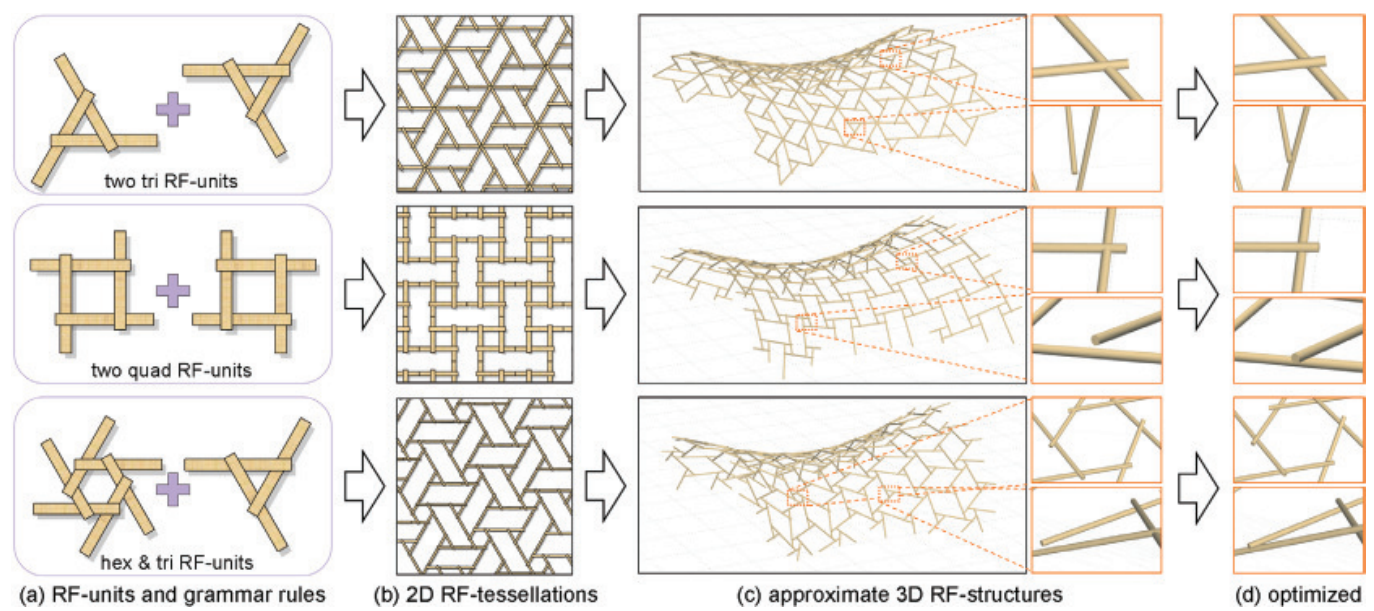

Figure 3: Overview: From basic RF-units, we tessellate the plane, map the tessellation to 3D, and then optimize the structure to be coherent.

Designing structures with a small number of RF-units is not an easy task, but manageable. Designing RF-structures that span over large domains is an intricate and complex task. We address the problem in two steps: we first build a grillage of RF-units in 2D and then lift it over a 3D guiding surface. The two steps are non-trivial. First, simply connecting RF-units is unlikely to form a coherent structure as the connection between them may contradict. We draw an analogy with the problem of tiling a plane with regular polygons, and develop a computational scheme to test whether a grillage of given RF-units forms a valid structure. Second, lifting an RF-grillage over a given surface is necessarily carried by a non-isometric mapping, which unavoidably introduces certain distortion. Our lifting is based on a conformal mapping followed by a relaxation process that respects both the angles among the RF-patterns and the lengths of their rods. Moreover, since rods are straight in 3D and contact with more than two others, conformal mapping can produce a good approximation of an RF-structure but cannot ensure collinear contact joints along each rod. Hence, we devise further a novel optimization model to ensure collinearity of contact joints along each rod, while preserving the geometric properties of the RF-structure.

Currently, architects mainly rely on simple approaches to design RF-grillages that are either manual and tedious [Gelez and Saby 2011], or too restrictive with very limited user controls [Brocato and Mondardini 2010; Thönnissen and Werenfels 2011]. Though computational methods for RF-structures have been used by the architectural community [Pugnale et al. 2011], the existing research still focuses more on the engineering issues, such as force analysis and stability of a given RF-structure, and not on the design.

Our work focuses on the design of large RF-structures. We introduce an interactive technique with which users can quickly sketch and formulate designs with extended number of RF-units, allowing them to easily manipulate, test, and preview a wide variety of coherent RF patterns with feasible geometric parameters. Our system can free the users from various engineering considerations, and allow them to focus on the aesthetic aspect of their designs.

\subsection{Related Work}

Background. For many centuries, reciprocal frames have been used in design and construction, e.g., the classical bridge sketches by Leonardo da Vinci, the roof of Nagasaki Castle in Japan, as well as Eskimo tents (see [Larsen 2008]). However, the term "reciprocal frame" was coined only in late 1980s by designer Graham Brown, and developed for constructing roundhouses with RF roofs.

There exists little computational support to design and construct
RF-unit based structures. Hence, most such realizations are restricted to small structures involving only a few RF-units. Such an approach does not generalize to larger structures due to various challenges involving where to place the RF-units, how to interconnect them, and how to realize a meaningful aesthetic design.

RF-structures in practice. RF-structures are fast gaining popularity for practical eco-friendly constructions as the RF-units are simple to prefabricate and reuse. However, at present there is little support to guide users discover feasible arrangement of RF-units. Hence, architects often manually experiment with different ways of assembling RF-units by testing physical mock-ups created using rods (e.g., in a scale of 1:5) [Chilton 2009; Gelez and Saby 2011]. Although such an approach gives full control over the design, form finding remains challenging while ensuring a valid arrangement of the RF-units. As a result even relatively simple designs can be tedious and time-consuming to mock-up [Gelez and Saby 2011].

Pugnale et al. [2011] stressed the need for computational tools for RF-based designs. Existing attention, however, is focused on handling engineering issues such as force analysis on the structural stability [Douthe and Baverel 2009; Kohlhammer and Kotnik 2010] and the fitting of rods to form a connected RF [Baverel et al. 2004; Parigi et al. 2012].

Although there have been recent attempts to support RF design, they are preliminary and offer only limited user controls. Brocato and Mondardini [2010] proposed a geometric method to design stone domes with extended number of RF-units, but their method supports only one class of RF patterns and offers a few parameters for user control. Thönnissen and Werenfels [2011] employed a Rhino-script to aid students to design RF-structures and arranged the RF-units over the cells obtained as the Delaunay triangulation of points on the input surface. However, since the point set can have arbitrary distribution, the resulting RF-structures can be rather irregular. Further, the users have little control on the RF patterns, and have no support to interactively preview and refine the designs.

Fabrication-aware form finding. In a more general context of architectural geometry, different methods have been proposed for geometric modeling with different fabrication constraints. Whiting et al. [2009] explored structural feasibility in the context of modeling masonry buildings. They proposed a gradient-based optimization method to search the parameter space in procedural models to generate stable buildings, and more recently [2012] introduced a stability metric and performed gradient-based optimization to modify geometry to achieve stability. Concurrent efforts [Fu et al. 2010; Eigensatz et al. 2010; Singh and Schaefer 2010] introduced cost- 
driven methods to analyze and optimize panel types and shapes towards cost-effective constructions of free-form surfaces. Yang et al. [2011] devised a computational framework to characterize, parameterize, and navigate non-linearly constrained shape spaces to access feasible designs that satisfy a given set of constraints.

Computational design tools. With growing focus on physically manufacturable objects, e.g., papercraft models [Mitani and Suzuki 2004; Li et al. 2010], plushie toys [Mori and Igarashi 2007], beadwork model [Igarashi et al. 2012], and interlocking 3D puzzles [Xin et al. 2011], there has been increased effort in developing computational design tools. Paczkowski et al. [2011] introduced the Insitu system that combines site photos, elevation data, and site plans to form a stroke-based terrain representation for guiding the design of architectural sketches. Umetani et al. [2012] proposed a design suggestion interface that employs a force analysis model to guide users to design valid shapes of furniture models under geometric and physical constraints.

In this work, we develop a computational tool to support design and realization of large RF-structures, which are difficult to conceive using physical mockup-based experimentation. In our tool, one can quickly sketch up an RF-structure by combining different RF-units, flexibly modify its appearance and pattern, as well as interactively experiment with different design parameters while the underlying optimization ensures connectivity and structural coherence.

\subsection{Overview}

Our interactive computational tool considers two aspects: aesthetic and coherency. To achieve the first, the tool creates RF-patterns with high self-similarities and symmetries. A basic set of building blocks (see Figure 3(a)), which we call RF-units, are offered to the user, but the user can design and customize them if he likes. These units are symmetric; in most cases, they have a rotation symmetry or reflection symmetry. By generating a tessellation of a plane (see Figure 3(b)), and further lifting it up to 3D, we can design RF-tessellations on surfaces and form large-scale structures with visually appealing self-similarity patterns (see Figure 3(c\&d)).

To guarantee that the RF-tessellation is coherent, the system first validates that a $2 \mathrm{D}$ tessellation can be generated from the RF-units specified by the user; then, it optimizes the 3D RF-structure to further ensure that the resulting grillage is coherently-connected in $3 \mathrm{D}$, that is, the original angles between the rods of the RF-units are preserved, and the rods are properly contacting in 3D space.

The main objective of our interactive tool is to allow the user to easily design and visualize large RF-structures for a given guiding surface. First the RF-structure is designed on a plane and then lifted to a surface in 3D (see Figure 3(b\&c)). This interactive design process starts by picking typically one or two RF-units and defining the connecting rules between the basic units (see Section 2). These can be regarded as very simple grammar rules. However, unlike common grammar systems, here the grammar should lead to a coherent tessellation of the plane. To guarantee that this is non-trivial. We validate the grammar by constructing a minimal cycle of RFunits, and analyzing its encoded configuration (see Section 3). We show that the RF-tessellation is the dual of known tessellations of the plane with regular polygons.

Once the RF-tessellation is formed on the plane, it can be regarded as a flat polygonal mesh, where the junctions between the rods are the mesh vertices. To lift the mesh to the surface while preserving the angles, we employ a conformal map (see Section 4). Since we do not map points but straight rods, we need to guarantee the collinearity of contact joints along every rod. Furthermore, we would also need to minimize the rods length distortion. Hence, we apply a relaxation process to trade isometry and approximation followed by an optimization process to guarantee the collinearity of contact joints along each rod while preserving the geometric properties of the RF-structure (see Section 5).

The generated RF-structure is a parametric model. The user can interactively modify its parameters, preview its appearance on a guiding surface, and easily experiment with different design variations.

\section{Defining and Connecting RF-Units}

Large RF-structures consist of a grillage of rods; however, they are designed as a two-level hierarchy. First, small RF-units are defined, and then aggregated into a large grid. The fundamental elements can be rods, beams, bars, or sticks. Hereafter, we will refer to them as rods. A reciprocal arrangement of at least three rods forms an $R F$-unit, which defines the building blocks of an RF-structure. There are four common approaches to physically construct an RFunit from rods, or in general to connect two intersecting rods in an RF-structure: notching, nailing, tying, and friction. In our work, we follow the common approach taken by architects [Larsen 2008] for constructing large-scale RF-units, and assume that they are nailed or tied (see Figure 2 (right)).

Rods, in general, have thickness, and thus the RF-units are nonplanar. To form an RF-unit, the rods are placed over one another in a closed circuit (as in Figure 2), forming a dome-like 3D geometry. However, it has been known [Parigi et al. 2012] that for creating free-form shapes, one may need some non-circular arrangements of the type shown in Figure 4 (right) (see also Figure 2 (right)).

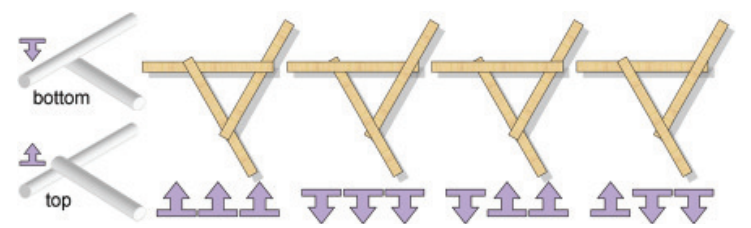

Figure 4: A three-rods RF-unit has four possible top/bottom arrangements.

The RF-unit can be treated as a parametric model with parameters such as rod thickness and vertical rise (see [Larsen 2008] for more detail). In our work, users can interactively edit the appearance of an RF-structure with the following parameters (see Figure 5): number of rods $(N)$; radius of inner circle $(R)$; rod length $(L)$; and clockwise and counter-clockwise spiraling (see Figure 5).
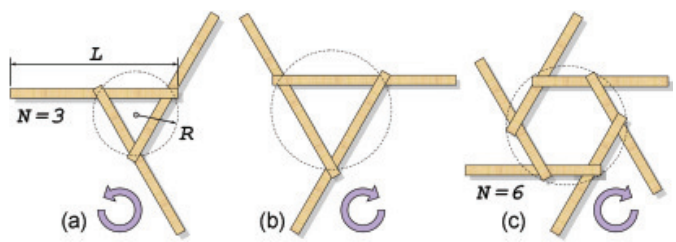

Figure 5: (a) parameter labels on an $N=3$ counter-clockwise $R F$-unit. (b) a clockwise RF-unit with a larger $R$ and $L$. (c) an $N=6$ clockwise RF-unit. Note: all rods are on top of others.

Our interface tool provides both clockwise and counter-clockwise RF-units for $N \in[3,6]$; one can control $L$ and $R$ to vary the appearance of an RF-unit. We do not explicitly provide RFunits with $N \geq 7$ since they are useful mainly for roundhouse domes with a single $N \geq 7 \mathrm{RF}$ unit rather than for making large $\mathrm{RF}$-structures as we focused in this work. However, motivated by Parigi and Pugnale [2012], who designed a bug-shaped 


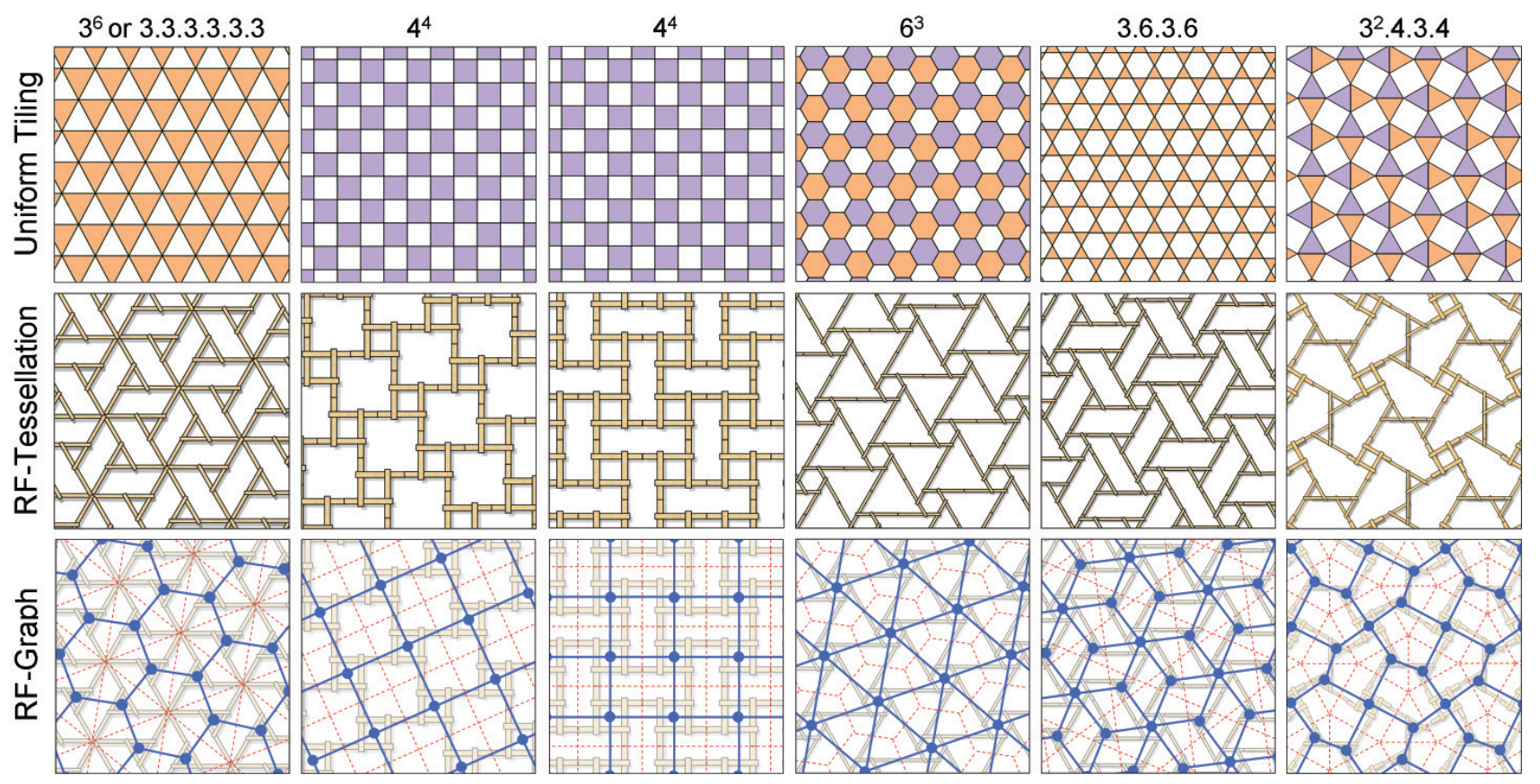

Figure 7: The relationship between plane tilings with congruent regular polygons (top row) and RF-tessellations, or RF-structures, (middle row): From an RF-structure, we construct an RF-graph (blue) and then its dual (red). Such dual is a plane tiling pattern implicitly associated with the RF-tessellation.

RF-unit that makes up RF-structures, our tool allows one to manipulate rods or edit an existing RF-unit to design new RF-units.

Since this work focuses on the design of aesthetic RF-structures, our tool deals only with regular RF-units whose rods are rotationally-symmetric. Hence, for the user-designed RF-unit, its external rods (those that connect to neighboring RF-units) have to be rotationally-symmetric.

The appearance of an RF-grillage is geometrically determined by three factors: (i) geometric parameters of the individual RF-unit (see Figure 5), (ii) the connections of RF-units, and (iii) the geometry of the guiding surface. For (ii), there are three types of connection: sharing a common rod, an endpoint-to-endpoint contact, and a T-join contact (see Figure 6). Note that the first type is the most common type, and we include the other two for completeness; the endpoints in the second type are contacting but not stacking. Our design tool offers all three types, and users can continuously shift from one type to another. Moreover, it allows adjacent RF-units share more than one contact (see top row in Figure 3(a\&b)).

To design an RF-structure, the user selects two RF-units, which can either be the same or not. Then, he connects them in the working canvas of our tool to define the way these two RF-units are connected. We regard such connection as a grammar rule, with which

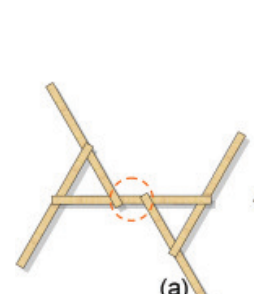

(a)

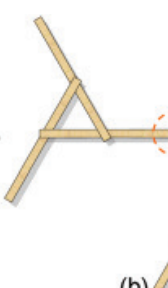

(b)

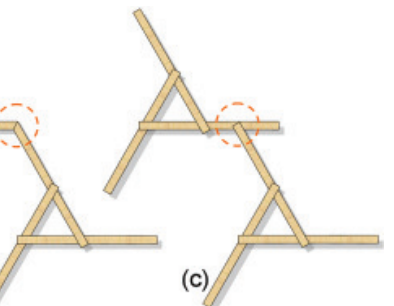

c)
Figure 6: Connecting two $R F$-units by (a) sharing a common rod, (b) an endpoint-to-endpoint contact, or (c) a T-join contact. the algorithm attempts to repeatedly apply to procedurally generate an RF-structure (see Figure 3(a) for examples). One grammar rule together with one RF-unit is sufficient to define this planar RFstructure, but users can also define more than one rule and/or employ more than one RF-unit to achieve a coherent RF-tessellation.

\section{RF-Tessellation of the Plane}

\subsection{Congruent Polygon Tessellation}

The tiling of the plane has been studied based on the theory of symmetry group and vertex transitivity [Grünbaum and Shephard 1986]. Stating the main idea in simpler words: a plane tiling by congruent regular polygons is uniform (or 1-uniform), if every vertex in the tiling is surrounded by exactly the same sequence of polygons.

Such a sequence of polygons can be encoded by considering the polygon degrees: 3 for triangles, 4 for squares, etc., and a superscript index for their multiplicity. This encoding is called a vertex configuration. See Figure 7 (top row) for five different tilings and their corresponding vertex configurations above. If a tiling involves only a single type of polygons (see the first four columns in the figure), it is called a regular tiling. It is known that there are only three regular tilings for congruent regular polygons.

The study of RF-structures is mainly in the architectural and engineering fields. We observed that there is a unique and direct connection between RF-tessellations and the tiling theory [GrÜnbaum and Shephard 1986]. More precisely, we found that
An RF-tessellation with rotationally-symmetric $R F$-units is structurally-equivalent (dual) to an edge-to-edge tiling by congruent regular polygons.

We illustrate this relationship with six different examples, shown in Figure 7: From an RF-tessellation, or an RF-structure (see Figure 7 (middle row)), we first construct a graph structure, which we call an $R F$-graph. This RF-graph considers RF-units as vertices (the blue 
dots in the last row) and their connections with adjacent RF-units as edges. By regarding an RF-graph as a mesh, we further construct its dual (the dashed red lines in the figure) by connecting the centroids between every pair of adjacent faces in the mesh. Such dual mesh is in fact the underlying plane tiling pattern (see again Figure 7 (top row)) implicitly associated with the RF-structure.

Based on this formulation, we say that the dual of an RF-tessellation is an edge-to-edge tiling by regular polygons, e.g., three-rods RFunits are dual of triangles, four-rods RF-units dual of squares, etc. This analogy allows analyzing the RF-tessellation by the knowledge of regular polygonal tiling.

The mapping between RF-tessellations and regular tilings is not one-to-one but many-to-one. See, for example, the second and third columns in Figure 7. Both RF-structures correspond to the same tiling, but their RF-units arrangement is different. In fact, taking into account the variation of the RF-unit parameters and their connection types, there are infinite number of ways (when considering continual parameters) to connect, say, four-rods RF-units, which are all mapped to the same tiling with squares.

On the other hand, starting from a plane tiling, such as the nontrivial one shown on top-right of Figure 7, we can pick the two corresponding RF-units, i.e., a three- and a four-rods RF-units, and construct an associated RF-tessellation, see the RF-structure in Figure 7 (middle right). It is worth noting that we are unaware of any existing RF-structure associated with this tessellation.

Linking RF-tessellations and the tiling theory is valuable beyond gaining insight about novel RF-structures, we in fact can further take advantages of the tiling theory: (i) to test whether the grammar rules from the users can form a coherent valid tiling, and in case it is, (ii) to position the RF-unit(s) within a tile, and (iii) to effortlessly generate an RF-tessellation, rather than exhaustively testing the validity of the rules, as we shall elaborate below.

\subsection{RF-Tessellation}

From tiling theory, we know that, after excluding a mirrored tiling, there are altogether eleven 1-uniform tilings [Grünbaum and Shephard 1986]; among them, eight involve polygons with degrees less than or equal to six. Their vertex configurations are $3^{6}, 4^{4}, 6^{3}$, $3^{4} .6,3.6 .3 .6,3^{3} .4^{2}, 3^{2} .4 .3 .4$, and 3.4.6.4. These configurations exhaust all valid plane tilings with regular polygons (from triangles to hexagons) because the angle sum at a vertex must be exactly equal to 360 degrees. In other words, in a valid tiling, every vertex corner must correspond to one of these configurations.

Based on the above, we can immediately test whether the given grammar rules lead to a coherent RF-tessellation. Our approach is to repeatedly apply the rules to concatenate a sequence of RF-units to test whether they complete a cycle.

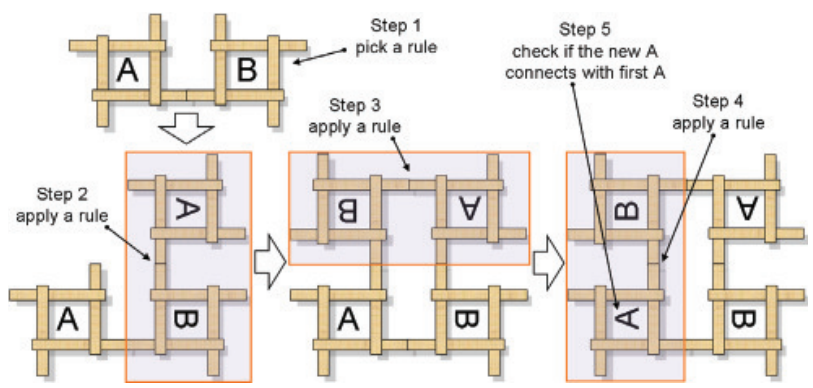

Figure 8: Iteratively applying a grammar rule to look for a cycle of RF-units, i.e., whether the last RF-unit gets back to the first.
This validity test is detailed as follows (see also Figure 8): Starting from a user-defined rule, or the seed RF-unit in it, we iteratively attach to it a new RF-unit by applying a grammar rule ${ }^{1}$. If the last RF-unit happens to connect back to the first one, we find a cycle of RF-units. If there are no loopbacks, the rules are said to be invalid. Since there are at most six polygons participating (i.e., six RF-units) in all the eight valid vertex configurations, we only need to build and test small sequences of up to seven RF-units, which amount to an exhaustive traversal of a small $k$-tree, where $k$ is the number of rules defined by the user.

For example, to generate the RF-structures shown in the first to fifth columns of Figure 7, only one grammar rule is sufficient since there is only one type of edges in the uniform tilings. However, two grammar rules are needed for the more complicated RF-structure shown on the rightmost column: one rule to connect the two three-rods RF-units and another to connect the threerods and four-rods RF-units (see the inset on the right).

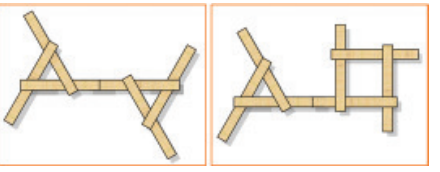

If we find a cycle of RF-units from the iterative process, we can use its encoded configuration to determine the corresponding tiling associated with the RF-structure. Having this underlying tiling structure, the dual RF-graph is readily available, over which the RF-units can be positioned to form the RF-tessellation (see Figure 7). Furthermore, when a user manipulates a grammar rule, the associated tiles can be interactively modified, and instantaneously reflected in the RF-tessellation, see more results in Section 6.

Lastly, it is worth noting that the validity test may discover more than one valid cycles in particular with multiple rules. For instance, given the two grammar rules shown previously in the inset figure, the rule on the left alone could form a cycle of six RF-units, and thus a $3^{6}$ tiling. Hence, if two or more cycles are discovered, users can choose which one to be used to form the RF-tessellation.

\section{Lifting the RF-Tessellation to 3D}

In this section we describe the procedure to map the RF-tessellation to a 3D guiding surface (Section 4.1) and to form an initial RFstructure in 3D (Section 4.2) to support interactive RF design.

\subsection{Mapping the RF-Tessellation to a Surface}

Recall that an RF-structure is a two-level hierarchy composed of RF-units and rods. To maintain its structural properties after lifting it up to $3 \mathrm{D}$, we have to preserve both the angles between contacting rods and the relative distances between adjacent RF-units. Unless the guiding surface is developable, an isometric mapping cannot exist, and we have to trade off between angle and length preservation. To maintain the structural symmetry, angle preservation has higher priority, and a conformal mapping is used. We use the ABF++ method [Sheffer et al. 2005] as a provably valid conformal parameterization with low length distortion.

The $\mathrm{ABF}++$ method parameterizes a 3D triangular mesh, denoted by $\mathcal{M}$, on the plane, which we denote by $m$ (see Figure 9(a)). After determining the $2 \mathrm{D}$ bounding box of $m$, our tool automatically translates and uniformly-scales $m$ to fit it in a unit square. We call this space, the RF-tessellation space, denoted by $\mathcal{S}$ (see Figure 9 (b)). To facilitate our discussion, we define $\mathcal{F}_{c}$ as the mapping

\footnotetext{
${ }^{1}$ When attaching a new RF-unit, such as the new A (above) to B in step 2 of Figure 8, we only need to consider B's rod that neighbors (clockwise) to the rod it shares with the previous A, but not the other rods of B. This applies to other steps as well, and is sufficient for testing if a loopback occurs.
} 


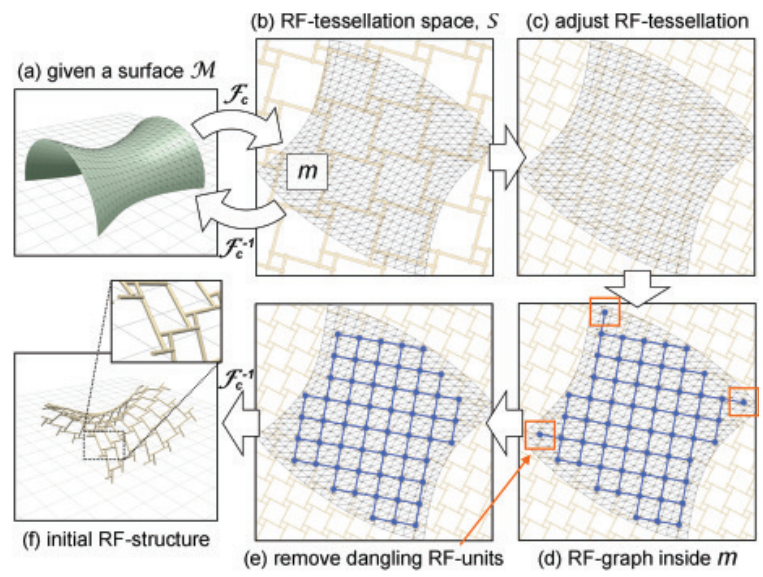

Figure 9: Procedure to lift an RF-tessellation to 3D space.

from $\mathcal{M}$ to $m$. The inverse mapping $\mathcal{F}_{c}^{-1}$ is defined using barycentric coordinates to interpolate the points mapped by $\mathcal{F}_{c}$; it will later be used to lift the RF-tessellation to the guiding surface.

The RF-tessellation is unbounded in 2D, so users can adjust its location, orientation, and scale, relative to $\mathcal{S}$, to specify its portion that is to be lifted to the guiding surface (see Figure 9(c)). By then, we can locate the RF-units within $m$ (see Figure 9(d)), and crop the RF-tessellation by $m$. To assist the users in this interactive adjustment, our tool renders the footprint of $\mathcal{M}$, i.e., $m$, on $\mathcal{S}$ to show the associated area covered by $\mathcal{M}$. Lastly, we remove also dangling RF-units with only a single neighbor, and connect the remaining RF-units to form a coherent RF-graph on $\mathcal{S}$ (see Figure 9(e)).

\subsection{Computing an approximate RF-structure in 3D}

Once a coherent RF-graph on $\mathcal{S}$ is formed, rods can be lifted to $3 \mathrm{D}$ by $\mathcal{F}_{c}^{-1}$ (see Figure 9(f)). However, the challenge here is that an RF-tessellation consists of straight rods, where each rod could contact with four others (see Figure 10 (left)). If we map points independently from $2 \mathrm{D}$ to $3 \mathrm{D}$ by $\mathcal{F}_{c}^{-1}$, the mapped rods are no longer straight in $3 \mathrm{D}$, and the mapped tessellation is no longer valid. Such lifting is non-trivial. The four mapped contact joints should be collinear in $3 D$ since rods in a valid RF-structure should be straight (see Figure 10 (right)). Thus, we first produce an approximate RFstructure with floating rods in $3 \mathrm{D}$, and later optimize this initial layout (rods position) to make it coherent (Section 5).

To produce an approximate RF-structure, we map rods with some special care to keep contacting rods close to each other. The details are as follows (see also Figure 11): For each RF-unit in the cropped RF-graph, we first locate the expected contact points between neighboring rods on $\mathcal{S}$. Then, we compute the mid-point between each pair of adjacent contact points, and map it to 3D by $\mathcal{F}_{c}^{-1}$. In addition, for each rod that is not shared by two RF-units, including those with the second and third connection types (see Fig-

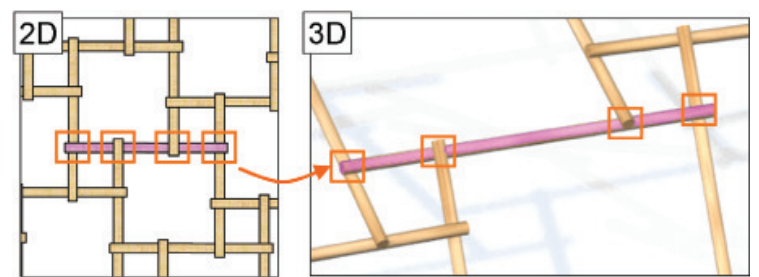

Figure 10: In a valid RF-structure, rods not only should be straight in $3 D$, but should also have collinear contact joints with others.

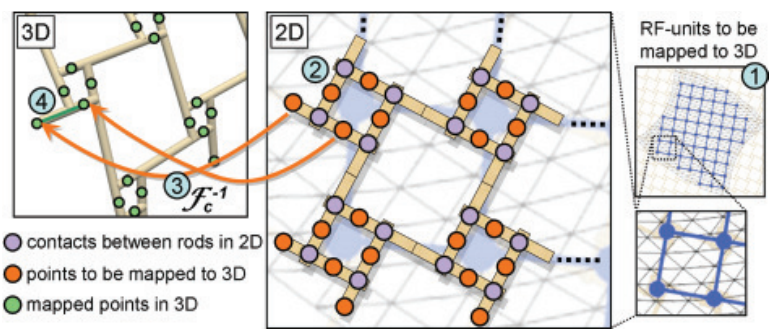

Figure 11: Initial placement of rods in $3 D$ by $\mathcal{F}_{c}^{-1}:$ 1) locate expected contact points (purple) between rods; 2) locate the related mid-points and endpoints (orange); 3) map these orange points from $\mathcal{S}$ to $3 D$ by $\mathcal{F}_{c}^{-1}$; and 4) connect the mapped points (green) correspondingly to layout the rods in $3 D$.

ure 6), and those on the RF-graph boundary, we map also its endpoint from $\mathcal{S}$ to $3 \mathrm{D}$. By connecting the mapped points in 3D correspondingly for each rod, we can layout the rods in 3D and get an approximate RF-structure (see Figure 11 (left)). Another advantage of this scheme is that the top/bottom rods arrangement in each RFunit can naturally follow the local surface curvature (see Figure 4). The RF-units in convex/concave regions will receive all-up/down arrangement, while those in near-planar regions will have a mixed rods arrangement. Note that like the example shown in Figure 2 (right), rods have to be tied in the physical assembly, especially for all-down/mixed arrangements in which rods are not self-supported.

The above lifting procedure takes a guiding surface and a 2D RFtessellation as its input, defines $\mathcal{F}_{c}^{-1}$, and directly generates a good approximation of an RF-structure, which enables an interactive preview. At this stage, the lifted structure is still imperfect. Some rods might penetrate, or slightly float above one another, rather than contacting (see Figure 3(c)). However, the user's focus at this stage is on the aesthetic aspects of the designs, exploring the design space, and interactively trying various parameters, rules, and mappings. For all the modifications, the user can get an instantaneous visual feedback (see supplementary video). With this interactive preview loop, users, for the first time, can flexibly try many different forms of putting up the RF-units on the same guiding surface, and freely explore the various design choices. See Section 6 for examples.

\section{Optimizing the RF-Structure in 3D}

There are two concerns to make the approximate RF-structure coherent in 3D. The first is about its geometry. We need to optimize the rods placement to meet the collinearity requirement described earlier. There are some recent solutions to the problem, but they either work with small-scaled models with a few RF-units [Baverel et al. 2004], or do not consider the tessellation symmetry [Parigi et al. 2012]. Compared to these existing solutions, our formulation for the optimization is novel; it can handle large RF-structures with extended number of RF-units, and considers also the structural symmetry and shape of the RF-units. Moreover, it is a two-level optimization that matches the RF hierarchy: (i) relax the RF-graph in 3D (Section 5.1), and (ii) optimize the rods positions (Section 5.2). The second concern is about the stability, where we use ANSYS, a professional software, to perform stress analysis on the optimized RF-structure (Section 5.3).

\subsection{Relaxation of the RF-graph in 3D}

The first level optimization is to reposition the RF-units in the 3D RF-graph, aiming at securing a better initial rods arrangement in $3 \mathrm{D}$. Our objective here is to displace the RF-graph vertices (centers of RF-units), such that the angles at each vertex and the relative 

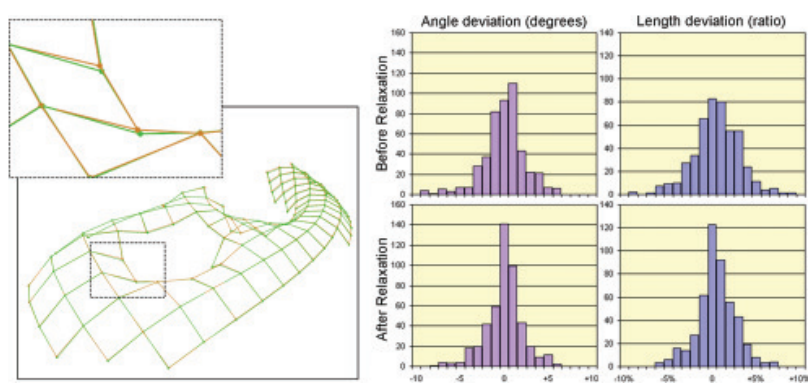

Figure 12: Left: RF-graphs (in 3D) before and after the relaxation are colored in orange and green, respectively; note that the optimization is local. Right: histograms of the corresponding angles and edge lengths deviations. The computation time here is 0.265 sec. on a desktop computer with $3.2 \mathrm{GHz} \mathrm{CPU}$ and $9 \mathrm{~GB}$ memory.

edge lengths match those in the corresponding 2D RF-tessellation. We achieve this by fixing one edge of the mesh and updating all the other vertices by the following minimization:

$\mathcal{V}^{*}=\underset{\mathcal{V}}{\operatorname{argmin}}\left[\sum_{\theta_{i}}\left(\cos \theta_{i}-\cos \theta_{i}^{0}\right)^{2}+\lambda \sum_{i, j}\left(\left\|P_{i}-P_{j}\right\|-l_{i j}\right)^{2}\right]$, where the first and second summation terms run over all angles $\theta_{i}$ and edges $P_{i} P_{j}$, respectively; $\mathcal{V}$ and $\mathcal{V}^{*}$ are the vertex sets in 3D (excluding the two vertices on the fixed edge) before and after the relaxation, respectively; $\theta_{i}^{0}$ and $l_{i j}$ are the ideal angles and lengths (from the RF-tessellation), respectively; and $\lambda$ is the tradeoff factor with default value 1.5. In our implementation, we linearize the minimization and iteratively solve for all vertices via a least-squares method, so there are no traversal orders. Moreover, we include a fidelity term to keep the vertices close to the surface. See Figure 12 for the results. Lastly, the relaxation result is interpreted as a $2 \mathrm{D}$ deformation of the RF-graph in the RF-tessellation space, say $\mathcal{D}$. With $\mathcal{V}^{*}$, we can employ $\mathcal{F}_{c}^{-1} \circ \mathcal{D}$ instead of $\mathcal{F}_{c}^{-1}$ to improve the rods arrangement in $3 \mathrm{D}$, using the scheme described in Figure 11.

\subsection{Optimization of Rods Positions in 3D}

The second level optimization is on the rods positions. To this end, we introduce three constraints as described below:

(i) Contact constraint. To simplify the formulation, we model rods as cylinders (diameter $w$ ). Thus, when two rods contact, the distance between their central axes (see Figure 13(a)) is $w$. Moreover, to fulfill the collinearity requirement (see again Figure 10), we model each rod, say rod $i$, with $A_{i}$ and $B_{i}$ as its endpoints in 3D, and $P_{i}(t)=A_{i}+t\left(B_{i}-A_{i}\right)$ as its central axis with parameter $t$. If rods $i$ and $j$ contact, we denote $P_{i}^{j}=P_{i}\left(t_{i}^{j}\right)$ and $P_{j}^{i}=P_{j}\left(t_{j}^{i}\right)$ as the contact joints on them, respectively, and $N_{i j}$ as the unit vector perpendicular to both $A_{i} B_{i}$ and $A_{j} B_{j}$. Note that $N_{i j}$ is a function of $A_{i}, B_{i}, A_{j}$, and $B_{j}$, and its direction is based on the top/down positioning between $P_{i}^{j}$ and $P_{j}^{i}$. Hence, the contact constraint is

$$
F_{1}\left(P_{i}^{j}, P_{j}^{i}\right) \triangleq P_{i}^{j}-P_{j}^{i}-w N_{i j}=0 .
$$

To cater for the second connection type (see Figure 6 (b)), we set $w=0$ because the related rods are contacting but not stacking.

(ii) Surface constraint. Second, we keep RF-units close to the surface by requiring each of their centroids to lie on the surface:

$$
F_{2}\left(V_{1}, \cdots, V_{m}\right) \triangleq \frac{V_{1}+V_{2}+\cdots+V_{m}}{m}-G=0,
$$

where $V_{i}$ are the rods endpoints in the inner side of the RF-units (with degree $m$ ) and $G$ is the surface point that corresponds to the node on the 3D RF-graph (see Figure 13(b)).
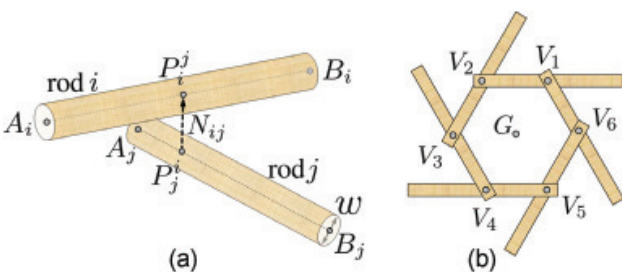

Figure 13: Notations used in the constraint definitions.

(iii) Conformality constraint. Third, for each RF-unit, we regularize each angle, i.e., $\angle V_{i-1} V_{i} V_{i+1}$, in the inner loop by

$$
F_{3}\left(V_{i-1}, V_{i}, V_{i+1}\right) \triangleq \frac{\left(V_{i-1}-V_{i}\right) \cdot\left(V_{i+1}-V_{i}\right)}{\left\|V_{i-1}-V_{i}\right\|\left\|V_{i+1}-V_{i}\right\|}-\cos \theta_{i}=0,
$$

where $\theta_{i}=(m-2) \pi / m$, and regularize each edge $V_{i} V_{i+1}$ by

$$
F_{4}\left(V_{i}, V_{i+1}\right) \triangleq\left\|V_{i+1}-V_{i}\right\|-l_{i}=0,
$$

where $l_{i}$ is the average length of all edges in the inner loop.

Objective function. We put together the above three constraints into a minimization problem:

$$
\begin{aligned}
\min _{\text {all rods }} & \alpha \sum_{i, j} F_{1}^{2}\left(P_{j}^{i}, P_{i}^{j}\right)+\beta \sum_{\left\{V_{i}\right\}} F_{2}^{2}\left(V_{1} V_{2} \cdots V_{m}\right) \\
+ & \gamma \sum_{i} F_{3}^{2}\left(V_{i-1}, V_{i}, V_{i+1}\right) \\
+ & \zeta \sum_{i} F_{4}^{2}\left(V_{i}, V_{i+1}\right)+\lambda \sum_{i}\left(P_{i}-\bar{P}_{i}\right)^{2}
\end{aligned}
$$

with positive tradeoff factors $\alpha, \beta, \gamma, \zeta$, and $\lambda$, whose values are by default $500,5,1,1$, and 1 , respectively. The fifth term is introduced as a fidelity term to constrain the rod endpoints $A_{i}$ and $B_{i}$ not to deviate too much from their positions, $\bar{A}_{i}$ and $\bar{B}_{i}$, in the previous iteration. Like the relaxation, we again linearize the minimization and solve it via a least-squares method. Once the rods are updated by the minimization, we determine new contact joints positions as the nearest point pairs on each pair of contacting rods. We iterate this process until convergence. See Figures 3(c\&d) for RF-structures before and after this optimization, Figure 14 for the corresponding histogram distributions of gap sizes between contacting rods, and Table 1 for the related computational performance.

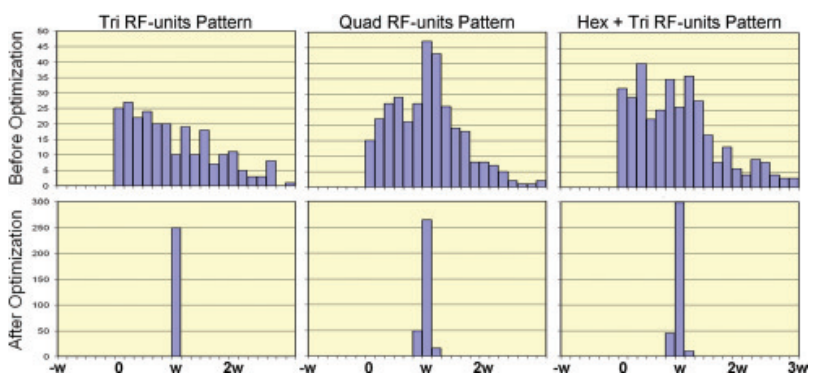

Figure 14: Histogram of gap sizes between contacting rods in the $R F$-structures shown in Figure 3(c\&d), top to bottom, correspondingly. Ideally, the gap sizes here should equal rod thickness (w).

Table 1: Time taken to optimize rods positions.

\begin{tabular}{|c|c|c|c|c|c|}
\hline \# RF-units & 9 & 20 & 42 & 92 & 191 \\
\hline \# rods & 24 & 49 & 97 & 205 & 413 \\
\hline time $(\mathrm{sec})$. & 0.029 & 0.186 & 1.318 & 12.132 & 98.658 \\
\hline
\end{tabular}



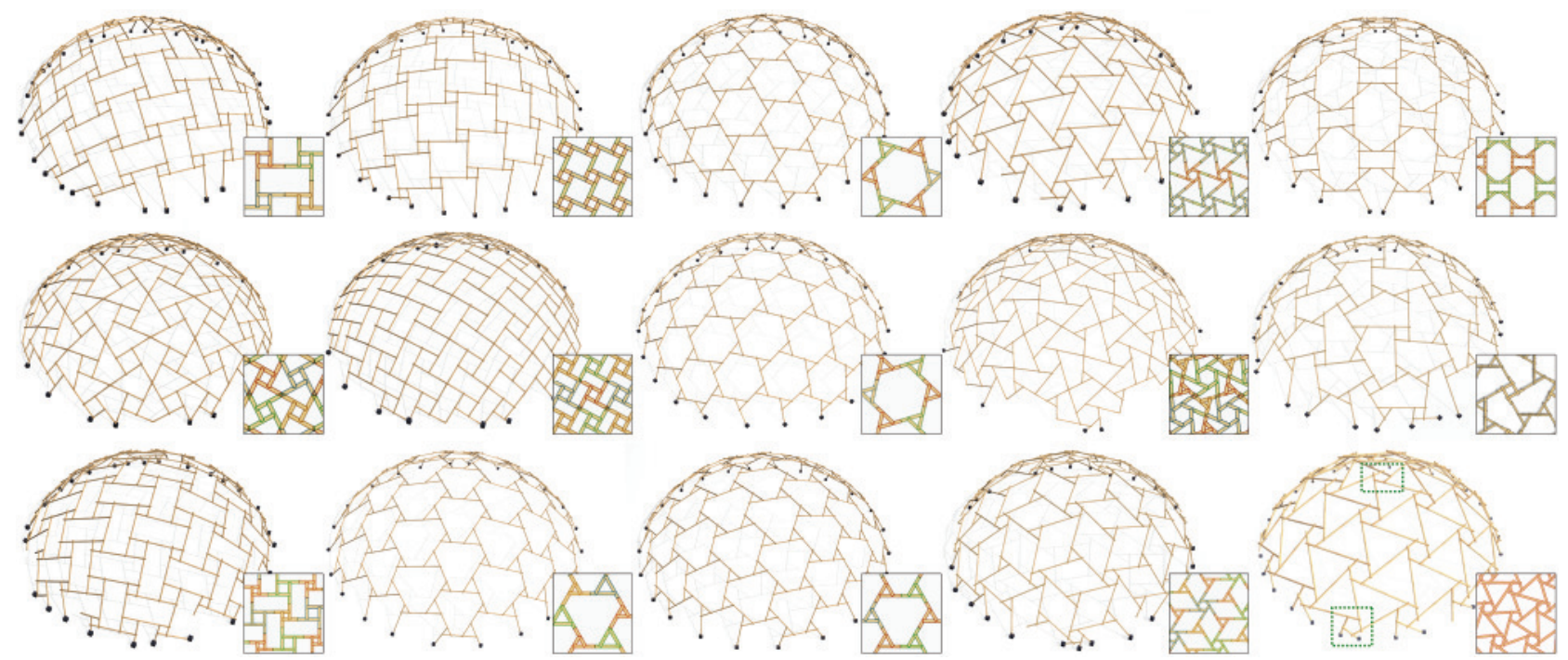

Figure 15: Our tool supports a wide variety of RF-tessellation patterns for designing RF-structures of different appearance.

\subsection{Stress analysis}

We employ ANSYS (Academic Research, Release 14.0), a finiteelement analysis package widely used in industry, to perform stress analysis on the optimized RF-structures. First, we interactively identify ground-supporting rods among the rods on the RF-structure boundary by thresholding; these rods are automatically adjusted to contact the ground and give support (see the black boxes in Figures 15 and 16). Then, our tool can automatically mesh and script the structure for input to ANSYS. In detail, we scale the RFstructure to around $10 \mathrm{~m}$, and use wood with density $800 \mathrm{~kg} / \mathrm{m}^{3}$ and Young's modulus $11 \mathrm{GPa}$ as the rods material. Typically, ANSYS takes around one to three seconds to compute, and in case of insufficient ground supports, it will refuse to produce outputs.

Figure 16 shows the von Mises stress plots on the two RF-structures that appeared in Figure 3. The smaller sub-figures show the stress on the rods under self-load. Now, if we put in extra loads (each with $100 \mathrm{~N}$ ), see the bottom row, we can compute the stress response of the rods, and evaluate if the stress distribution is balanced subject to the loading. With this visualization, the user can get feedback to change the design, for example, adding more supports in the design, or revising the design to make the structure more balanced.

\section{Results}

Our tool is implemented using $\mathrm{C}++$ and OpenGL, and it consists of two main panels (see Figure 17): (i) RF Pattern Editor, for composing and editing RF-units and grammar rules; and (ii) RF Creator,

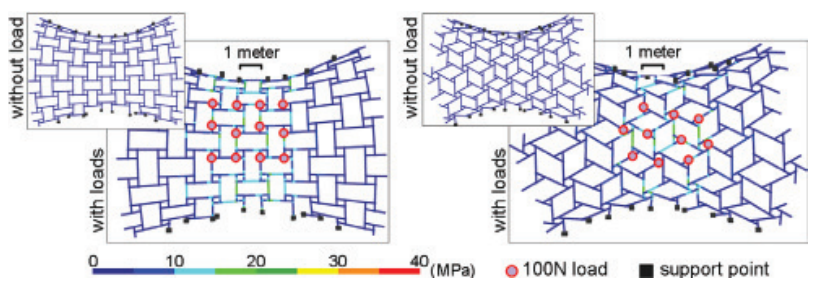

Figure 16: von Mises stress plots. The colors indicate the amount of stress on the rods; note the maximum stress for bamboo and pine wood are around $350 \mathrm{MPa}$ and $40 \mathrm{MPa}$, respectively.

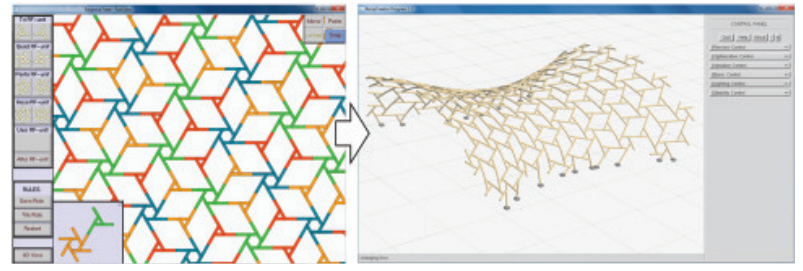

Figure 17: RF Pattern Editor (left) and RF Creator (right).

for making RF designs with interactive editing, for performing the optimization, and for communicating with ANSYS. As for the twolevel optimizations, we formulate them using least-squares fit, and solve them with the GNU Scientific Library [Galassi et al. 2009].

RF-designs. Our tool supports a wide variety of RF-patterns, and their variations. Figure 15 shows fifteen of them on a spherical dome. Note that some patterns, such as the three patterns on the first column, actually belong to the same pattern class. Our interactive tool allows us to continuously shift between them (see supplementary video). Note also the last column, where we present the userdefined bug-shaped RF-unit (from Section 2) and the non-trivial pattern below it (from Figure 7 (top-right)). On the other hand, we can design RF-structures over guiding surfaces of many different shapes. See Figure 18 (a-i) for SUPER BUG, TRAINSTATION, ELLIPSOID, IGLOO, SEASHELL, PEANUT, HUT, BRIDGE, and VASE.

Extension. We extend our tool to construct the classical reciprocal design known as the Da Vinci dome. In detail, we implement a
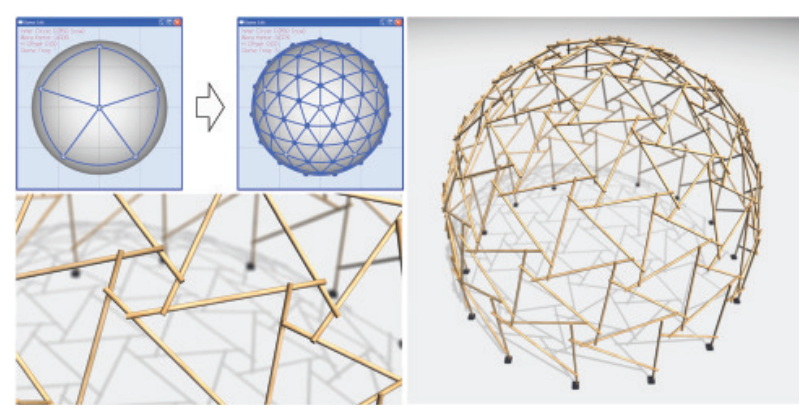

Figure 19: The Da Vinci dome designed by our tool. 


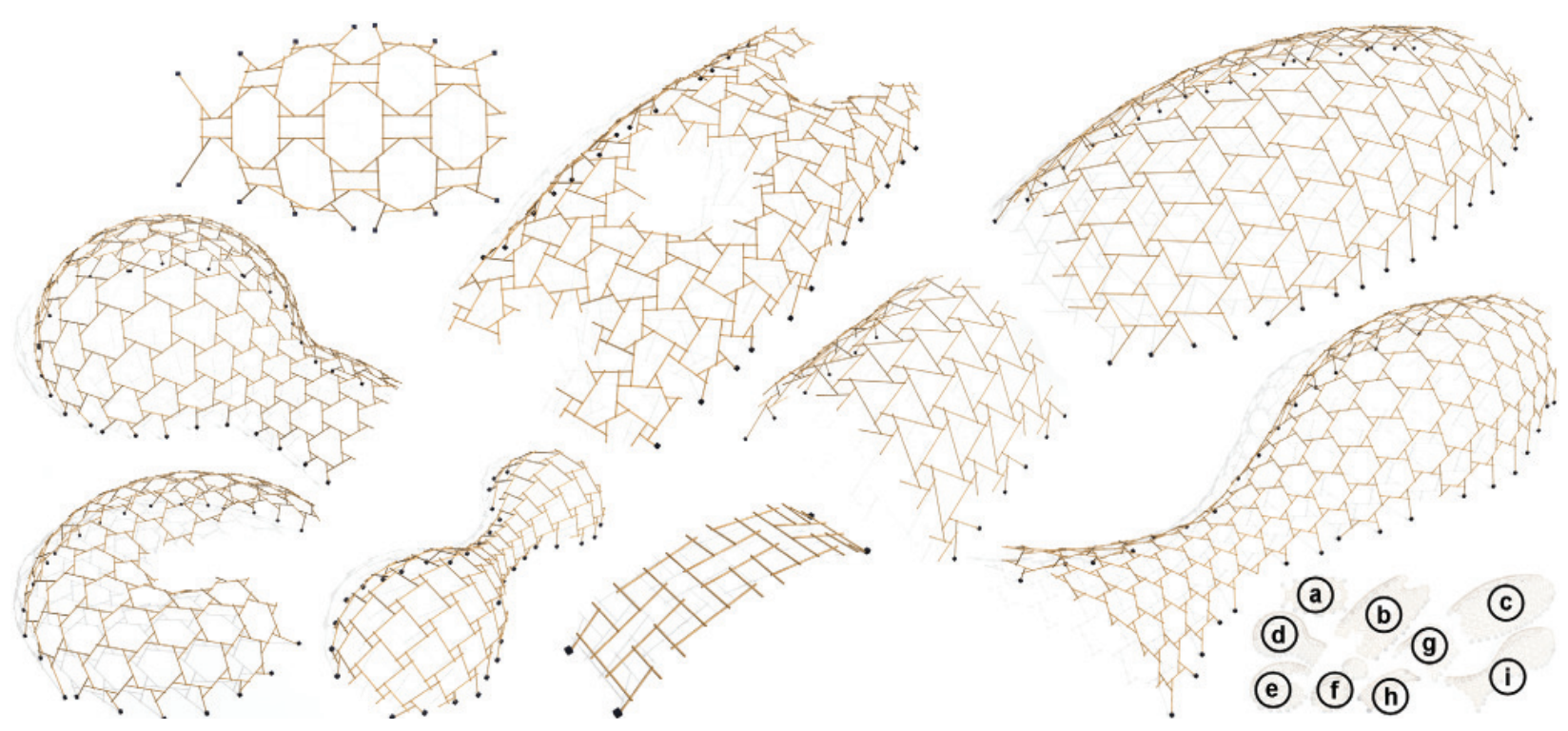

Figure 18: A collection of RF-structures designed by our tool (in order of minutes) using guiding surfaces of different shapes.

spherical tessellation plugin (see Figure 19 (top-left)) and connect it to $R F$ Creator; by this, we can interactively adjust the spherical tessellation frequency, apply a spherical warping on the pattern, and make use of a spherical mapping (instead of a conformal map) to generate RF-domes. In particular, this result shows that our approach is general and compatible with spherical embedding.

Physical construction. Our tool also offers interactive supports to aid the physical construction. The user can click on individual rod in $R F$ Creator to obtain its contacts information (angles and distances measurement) with the neighbors, and see a top-down map of ground-supporting contacts. See Figure $20(a \& b)$ for these visual aids and (c-h) for models we assembled with $3 \mathrm{~mm}$-thick wooden sticks. Note also that we can load (book) on top of the SUPER BUG.

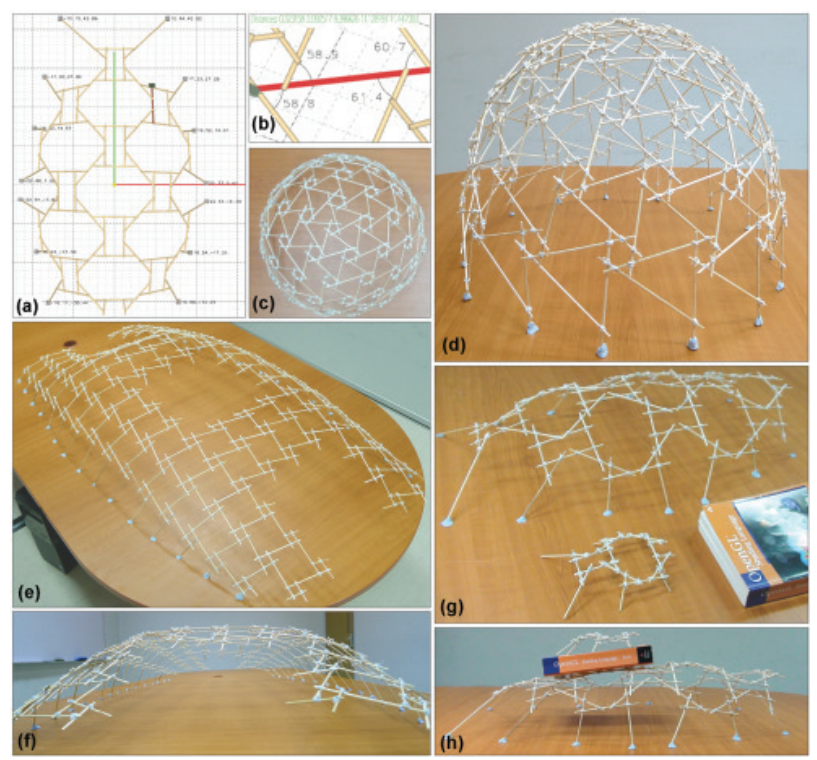

Figure 20: Physical construction: (a\&b) visual aids; $(c \& d)$ the Da Vinci Dome (195 sticks); (e\&f) TRAINSTATION in Figure 1 (267 sticks); and ( $g \& h)$ SMALL BUG and SUPER BUG (19 and 77 sticks).

\section{Conclusions}

This paper presents a novel computational approach to aid the design of large reciprocal frame structures. Our first contribution is on drawing an analogy between RF-structures and the plane tiling, with which we can verify and construct RF-tessellations defined with simple grammar rules. Apart from plane tiling, we also demonstrate the feasibility of our approach with spherical tessellation pattern through the Da Vinci dome example. Our second contribution is on applying a conformal map to lift the 2D RF-tessellation to 3D and computing an approximate RF-structure that enables interactive design. Since this procedure is performed in real-time, users can interactively explore many different RFpatterns, as well as their variations, on the same guiding surface, and focus on the aesthetic aspect of their designs. Thirdly, we devise also a novel optimization method to address the geometric form finding problem. We consider not only the collinear contact constraint, but also the geometric properties of the RF-structure to maintain its symmetric patterns. Finally, we demonstrate the capability of our tool through a collection of RF designs: a wide variety of RF-patterns and RF-structures, the classical Da Vinci dome, and several physical models assembled with the aid of our tool. With this tool, we also succeed in creating some interesting new RF-patterns that we did not encounter in the literature before, e.g., the first two and the last one patterns in the second row of Figure 15.

Limitations. First, our tool considers only the basic type of uniform tilings, but not general edge-to-edge tilings. Second, in regions of high curvature, the ideal angles in 2D are not realizable due to Gaussian curvature constraints in 3D; thus, our system cannot ensure RF regularity and rods contacts (see Figure 21 (left)). Third, we cannot place RF-units properly on narrow surface regions
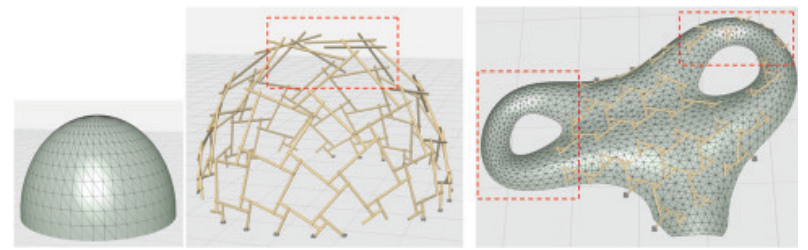

Figure 21: Failure cases. Left: RF-units on relatively high curvature regions. Right: missing RF-units on narrow regions. 
(see Figure 21 (right)). Lastly, we explore only regular RF units and one class of spherical tessellation.

Future work. We are interested in studying other forms of structural tilings on surfaces in 3D. Moreover, we also plan to study computational methods to determine the assembly order of RFstructures with Charlie bars to provide support during the intermediate construction stages. Other than by continuous editing of RF-units, we would like to explore alternative methods to design RF patterns, say, by selecting from a prepared pattern list. Another interesting direction will be to use the FEM simulator to guide form finding [Whiting et al. 2012; Umetani et al. 2012] in case when the current RF structure is not self-supporting under heavy load.

Acknowledgements. We thank anonymous reviewers for the various constructive comments, Yiorgos Chrysanthou for comments on our paper draft, Michael Brown for voice over, William Lai and Max Lim for help on 3D Studio Max, Yiyu Cai for sharing ANSYS, and Yongliang Yang for providing some of the reference 3D models. This work is supported in part by the Singapore MOE Tier-2 grant (MOE2011-T2-2-041), and the Israel Science Foundation.

\section{References}

Baverel, O., Nooshin, H., and Kuroiwa, Y. 2004. Configuration processing of nexorades using genetic algorithms. Jour. of the Intl. Assoc. for Shell and Spatial Structures 45, 2, 99-108.

Bertin, V. 2001. Variations of lever beam structures. In Proceedings of Conference on Growth and Form: The Engineering of Nature, School of Architecture, University of Waterloo.

Brocato, M., AND Mondardini, L. 2010. Geometric methods and computational mechanics for the design of stone domes based on Abeille's bond. In Advances in Architectural Geometry, Springer, 149-162.

Chilton, J. 1995. History of timber structures, Lecture E1. In STEP 2, Timber Engineering. STEP 2, Timber Engineering 2, E1-E13.

Chilton, J. 2009. Development of timber reciprocal frame structures in the UK. In Proceedings of IASS Symposium 2009: Evolution and trends in design, analysis and construction of shell and spatial structures, 1877-1884.

Douthe, C., AND Baverel, O. 2009. Design of nexorades or reciprocal frame systems with the dynamic relaxation method. Computers \& Structures 87, 21 (Nov.), 1296-1307.

Eigensatz, M., Kilian, M., Schiftner, A., Mitra, N., Pottmann, H., And Pauly, M. 2010. Paneling architectural freeform surfaces. ACM Tran. on Graphics (SIGGRAPH) 29, 4, 45:1-45:10.

Fu, C.-W., LAI, C.-F., He, Y., AND COHEN-Or, D. 2010. K-set tileable surfaces. ACM Tran. on Graphics (SIGGRAPH) 29, 4, 44:1-44:6.

Galassi, M., Davies, J., Theiler, J., Gough, B., Jungman, G., Alken, P., Booth, M., And Rossi, F. 2009. GNU Scientific Library Reference Manual. 3rd Ed., ISBN 0954612078.

Gelez, S., AND SABy, V. 2011. Nexorades, facing an emergency situation. Intl. Jour. of Space Structures 26, 4 (Nov.), 359-362.

Grünbaum, B., And Shephard, G. C. 1986. Tilings and patterns. W. H. Freeman \& Co.

IgARAShi, Y., IgARAShi, T., AND Mitani, J. 2012. Beady: interactive beadwork design and construction. ACM Tran. on Graphics (SIGGRAPH) 31, 4, 49:1-49:9.
Kohlhammer, T., And Kotnik, T. 2010. Systemic behaviour of plane reciprocal frame structures. Structural Engineering Intl. $21,1,80-86$.

LARSEN, O. P. 2008. Reciprocal Frame Structures. Elsevier Science and Technology.

Li, X.-Y., Shen, C.-H., Huang, S.-S., Ju, T., AND Hu, S.-M. 2010. Popup: automatic paper architectures from 3D models. ACM Tran. on Graphics (SIGGRAPH) 29, 4, 111:1-111:9.

Mitani, J., AND SUZUKI, H. 2004. Making papercraft toys from meshes using strip-based approximate unfolding. ACM Tran. on Graphics (SIGGRAPH) 23, 3, 259-263.

MORI, Y., AND IGARASHI, T. 2007. Plushie: an interactive design system for plush toys. ACM Tran. on Graphics (SIGGRAPH) 26, 3, $45: 1-45: 8$.

PaczKowski, P., Kim, M. H., Morvan, Y., Dorsey, J., RushMEIER, H., AND O'Sullivan, C. 2011. Insitu: sketching architectural designs in context. ACM Tran. on Graphics (SIGGRAPH ASIA) 30, 6, 182:1-182:10.

Parigi, D., And Pugnale, A. 2012. Three-dimensional reciprocal structures: morphology, concepts, generative rules. In IASS-APCS Proc. from spatial structures to space structures.

Parigi, D., KirkeGaard, P. H., and Sassone, M. 2012. Hybrid optimization in the design of reciprocal structures. In Proceedings of the IASS Symposium 2012: from spatial structures to spaces structures. 8 pages.

Pugnale, A., Parigi, D., Kirkegaard, P. H., and Sassone, M. 2011. The principle of structural reciprocity: history, properties and design issues. In IASS: Intl. Conference on Space Structures, 414-421.

Sheffer, A., LÉvy, B., Mogilnitsky, M., AND BoGOMYAKOV, A. 2005. ABF++: Fast and robust angle based flattening. ACM Tran. on Graphics 24, 2 (Apr.), 311-330.

Singh, M., AND SCHAEFER, S. 2010. Triangle surfaces with discrete equivalence classes. ACM Tran. on Graphics (SIGGRAPH) 29, 46:1-46:7.

ThÖNnissen, U., AND WeRENFELS, N. 2011. Reciprocal frames - teaching experiences. Intl. Jour. Of Space Structures 26, 4, 369-372. (Rhino-script developed by Prof. Annette Spiro).

Umetani, N., Igarashi, T., And Mitra, N. 2012. Guided exploration of physically valid shapes for furniture design. $A C M$ Tran. on Graphics (SIGGRAPH) 31, 4, 86:1-86:11.

Whiting, E., Ochsendorf, J., AND DuRAnd, F. 2009. Procedural modeling of structurally-sound masonry buildings. ACM Tran. on Graphics (SIGGRAPH ASIA) 28, 5, 112:1-112:9.

Whiting, E., Shin, H., WANG, R., OCHSENDORF, J., AND DURAND, F. 2012. Structural optimization of 3D masonry buildings. ACM Tran. on Graphics (SIGGRAPH ASIA) 31, 6, 159:1159:11.

Xin, S.-Q., LAI, C.-F., Fu, C.-W., Wong, T.-T., He, Y., AND COHEN-OR, D. 2011. Making burr puzzles from 3D models. ACM Tran. on Graphics (SIGGRAPH) 30, 4, 97:1-97:8.

YAng, Y.-L., YAng, Y.-J., PotTmann, H., AND Mitra, N. J. 2011. Shape space exploration of constrained meshes. ACM Tran. on Graphics (SIGGRAPH ASIA) 30, 6, 124:1-124:10. 